\title{
L'insertion professionnelle au prisme des relations entre parcours de formation et emploi. Le cas des diplômé.e.s de la faculté des sciences du sport de Strasbourg (2010-2014)
}

Professional integration through the prism of relations between training paths and employment. An example at the Faculty of Sports Sciences of Strasbourg (2010-2014)

Titre court : Du parcours de formation en STAPS à l'emploi From sport sciences training paths to employment

Sandrine Knobé ${ }^{1}$, ingénieure de recherche, Laboratoire Sport et sciences sociales (UR1342 ; E3S), Université de Strasbourg

Lilian Pichot ${ }^{2}$, maître de conférences, Faculté des sciences du sport et Laboratoire Sport et sciences sociales (UR1342; E3S), Université de Strasbourg

Anne Benoit ${ }^{3}$, ingénieure d'études, Faculté des sciences du sport et Laboratoire Sport et sciences sociales (UR1342; E3S), Université de Strasbourg

\section{Résumé}

S'appuyant sur une enquête quantitative et sur des entretiens semi-directifs réalisés avec des diplômé.e.s en sciences du sport (2010 à 2014) possédant un niveau de qualification bac +2 à $\mathrm{bac}+5$, la recherche se focalise sur les liens entre parcours de formation, insertion professionnelle et emploi occupé. Le parcours de formation et ses variations, d'un point de vue objectif et subjectif, sont questionnés comme élément clé de l'insertion professionnelle des diplômé.e.s, et en particulier de l'accès au premier emploi. Notre recherche met en évidence des situations d'emploi contrastées selon les niveaux de diplôme et les spécialités de formation, mais aussi «bricolées », du fait de parcours de formation rarement linéaires mêlant diplômes et expériences de préprofessionnalisation.

Mots-clés : insertion professionnelle, formation, diplômé.e.s, STAPS, emploi, parcours

\section{Professional integration through the prism of relations between training paths and employment. An example at the Faculty of Sports Sciences of Strasbourg (2010-2014)}

\begin{abstract}
Based on a quantitative survey and on semi-directive interviews conducted with graduates in sports sciences (2010 to 2014) with a level of qualification from 2 until 5 years after the bachelor, the research focuses on the links between training, professional integration and employment. The training path and its variations, from an objective and subjective point of view, are questioned as a key element of the professional integration of graduates, and in particular of the access to the first job. Our research highlights contrasted employment situations according to degree levels and training specialities, but also "tinkered" situations, due to rarely linear training pathways mixing diplomas and pre-professionalization experiences.
\end{abstract}

Keywords: professional integration, curricula, graduates, sport sciences, job, pathways

\footnotetext{
${ }^{1}$ Thèmes de recherche : insertion professionnelle ; employabilité ; sciences du sport. knobe@unistra.fr

${ }^{2}$ Thèmes de recherche : insertion professionnelle; employabilité ; sciences du sport. pichot@unistra.fr

${ }^{3}$ Thèmes de recherche : insertion professionnelle; employabilité ; sciences du sport. anne.benoit@unistra.fr
} 


\section{Introduction}

La question préoccupante de l'emploi des jeunes diplômé.e.s interpelle la place et la responsabilité des systèmes d'enseignement et de formation censés délivrer des diplômes, des qualifications facilitant l'accès à l'emploi. En France, le devenir professionnel des jeunes diplômé.e.s en STAPS (Sciences et Techniques des Activités Physiques et Sportives) se pose avec acuité, au regard de l'attractivité de la filière (18 672 étudiant.e.s entrant en première année en 2018 contre 7229 en 2008 - MESRI, 2019). Dans le même temps, les emplois relevant du domaine du sport, depuis le début des années 2000, restent estimés peu ou prou à $300000^{4}$, ordre de grandeur relativement stable. Des données précises et actualisées existent pour les emplois d'éducateur.rice.s sportif.ive.s au nombre de 63700 exerçant dans le secteur des activités sportives dans les clubs et les centres de fitness (Céreq Enquêtes, 2018) ${ }^{5}$. Par ailleurs, le commerce d'articles de sport ${ }^{6}$ emploie quelques 46500 salarié.e.s, chiffre stable depuis une vingtaine d'années.

Non sans lien avec la massification de l'enseignement supérieur et l'arrivée démographique de jeunes diplômé.e.s, la concurrence pour l'emploi et la sélectivité accrue du marché du travail ont entraîné un allongement, une incertitude et une complexification de la phase d'insertion professionnelle des jeunes (Arliaud \& Eckert, 2002). Le constat de la dissociation au moins partielle entre la sortie des études et l'entrée dans la vie active est admis (Dubar, 1999, 2001) et la relation entre le système d'enseignement et le système d'emploi n'est pas régie par une « adéquation formation-emploi » (Tanguy, 1986 ; Nicole-Drancourt \& Roulleau-Berger, 1995). Dès lors, il importe de comprendre comment cet espace postscolaire de transition entre études et emplois est structuré par des jeux complexes d'acteurs sociaux qui se déploient dans des contextes historiques et institutionnels déterminés. Ayant une autonomie relative, le marché de l'emploi dans le sport possède ses propres caractéristiques, ses organisations, ses normes de fonctionnement et des relations complexes, des codes qui lui sont spécifiques (Lima \& Mossé, 2010) et qui conduisent à développer des engagements de socialisation reconnus et valorisés.

En regard de cet univers particulier, la transition entre sortie des études et insertion professionnelle nécessite de mieux comprendre les liens entre parcours de formation et emploi occupé. Le parcours de formation est ainsi interrogé comme élément préparant l'insertion professionnelle des diplômé.e.s, en tenant compte autant des caractéristiques objectives de leurs parcours que de leurs perceptions subjectives.

\section{I - Du parcours de formation à l'emploi : contribution relative et variations}

La notion d'insertion professionnelle relève d'une construction sociale au cœur de laquelle se rencontrent, se confrontent, s'accordent ou non diverses catégories d'acteurs - les diplômé.e.s, les employeurs et les formateur.rice.s - et se construisent des grilles de lecture fondées sur des appréciations individuelles qui structurent des normes de fonctionnement et des pratiques en termes de recrutement et de recherche d'emploi. Indiscutablement protéiforme (Verdier \& Vultur, 2016), l'insertion professionnelle a longtemps été analysée comme un produit déterminé par la manière dont ont été construits les systèmes d'emploi et les dispositifs d'action publique (Silvestre, 1986), ou pour le dire autrement comme le processus par lequel un individu atteint une position stabilisée dans le système d'emploi (Vernières, 1997). Mais la compréhension de

\footnotetext{
${ }^{4}$ Ministère de la Ville, de la Jeunesse et des Sports, «Le poids économique du sport en 2013 », bulletin de statistiques et d'études, Mission des Études, de l'Observation et des Statistiques, Stat-info, n ${ }^{\circ} 16-3$, août 2016 ; «Les chiffres-clés du sport », Mission des Études, de l’Observation et des Statistiques, septembre 2015.

5 Dont 21000 non salarié.e.s.

${ }^{6}$ Relevant du code APE 47.64Z «Commerce de détail d'articles de sport en magasin spécialisé » dans la nomenclature des activités françaises.
} 
l'accès à l'emploi passe par d'autres voies de lecture que ne le subodore le modèle linéaire fondé sur la relation causale entre l'espace-temps de la formation et le marché du travail.

Ainsi la dimension processuelle qui invite à privilégier les configurations d'acteurs, les médiations sociales et les expériences individuelles nous conduit à être attentif aux séquences alternant temps de formation et temps d'expériences professionnalisantes propices à l'acquisition de compétences spécifiques. Dans cette direction, le parcours de formation (Pollien, 2010) revêtant un caractère préprofessionnel (Cahuzac \& Giret, 2001) nous semble devoir être considéré comme une catégorie d'analyse montante. Il peut être défini comme une ressource permettant de se prémunir individuellement contre les aléas du marché du travail et équipant les individus pour le marché (Gautié, 1993 ; Mériaux \& Verdier, 2009). Les variations observées sur les parcours de formation ont partie liée avec la multi-possession de diplômes, les arrêts et reprises d'études, les conditions d'accès à l'emploi dans un secteur professionnel donné. Les événements et les expériences qui jalonnent les parcours de formation individuels peuvent produire des effets sur l'accès à l'emploi et colorer les appréciations des diplômé.e.s, d'autant plus qu'ils renvoient aussi à des compétences non scolaires qui ne sont pas toujours formalisées et dont le seul diplôme ne rend pas compte (Tenret, 2011). Par conséquent, l'objectivation des parcours de formation incite à tenir compte des subjectivités (Ertul et al., 2014), c'est-à-dire des sentiments rétrospectifs exprimés par les individus à propos de leur place sur le marché du travail, de leur situation d'emploi (Vultur \& Trottier, 2010) et de la contribution relative de leur parcours de formation à la situation d'emploi vécue.

L'observation de l'insertion professionnelle des diplômé.e.s donne à voir des modalités d'accès à l'emploi et des situations d'emploi variées, dont certaines semblent davantage " bricolées » (cf. infra). Ainsi, notre objectif est de comprendre ces liens entre parcours de formation et insertion professionnelle, en portant une attention particulière à l'interprétation des tensions ou des décalages plus ou moins importants attestés objectivement et/ou ressentis par les diplômé.e.s. Dans quelles mesures les situations d'emploi «bricolées", révélant des tâtonnements, des moments d'hésitation, des bifurcations, sont liées au parcours de formation, aux diplômes obtenus et aux influences périphériques provenant de diverses socialisations (sportive, scolaire, familiale, etc.)?

\section{II - Une enquête quantitative et qualitative sur le devenir des diplômé.e.s en sciences du sport}

Pour tenter d'appréhender la complexité des parcours de formation, nous avons eu recours à une combinaison de techniques de recueil de données quantitatives et qualitatives (Mahlaoui, 2016). Une première enquête par questionnaire réalisée de mars à juin 2016 a donné lieu au traitement de données des réponses de 446 diplômé.e.s de la Faculté des sciences du sport de Strasbourg entre 2010 et 2014 (voir tableau 1). Le questionnaire a été adressé aux diplômé.e.s des formations proposées par cette faculté (voir figure 1), soit un total de 864 diplômé.e.s enquêté.e.s dont les coordonnées ont été extraites d'APOGEE.

En dehors des caractéristiques identitaires (âge, sexe, diplômes possédés), le questionnaire portait sur les items suivants : l'emploi occupé au $1^{\text {er }}$ janvier 2016 ; 1'accès au premier emploi et notamment le lien entre ce dernier et l'éventuel stage de fin d'étude ; le projet professionnel initial (lors de l'entrée en formation) ; les opinions relatives aux conditions d'accès au premier emploi, à l'adéquation entre le domaine de formation ainsi que le niveau de diplôme et l'emploi occupé, et l'utilité de la possession de plusieurs diplômes. Le questionnaire comprenait à la fois des questions fermées à choix multiples et des questions ouvertes. 


\section{Tableau 1}

Échantillon

\begin{tabular}{lcc}
\hline $\begin{array}{c}\text { Formations STAPS par } \\
\text { niveau de diplôme }\end{array}$ & $\begin{array}{c}\text { Nombre de diplômé.e.s } \\
\text { enquêté.e.s }\end{array}$ & $\begin{array}{c}\text { Nombre de répondant.e.s } \\
\text { (en \% des enquêté.e.s) }\end{array}$ \\
\hline Deust & 245 & $121(49.4 \%)$ \\
\hline Licence professionnelle & 38 & $36(94.7 \%)$ \\
\hline Licence $(*)$ & 279 & $70(25.1 \%)$ \\
\hline Master & 302 & $219(72.5 \%)$ \\
\hline TOTAL & 864 & $446(51.6 \%)$ \\
\hline
\end{tabular}

Table 1

Sample

* Nous avons extrait de la base de sondage les diplômé.e.s de licence en poursuite d'études au sein de la faculté des sciences du sport de Strasbourg.

Cette enquête, administrée en ligne et complétée par des relances téléphoniques, a été renseignée par $51,6 \%$ des diplômé.e.s de la période, dont un peu plus de femmes ${ }^{7}$. Malheureusement, du fait de l'absence d'informations préalables relatives aux diplômé.e.s, nous n'avons pas pu mener d'autres comparaisons liées à la structure respective de chacune des bases, diplômé.e.s et répondant.e.s. Les données quantitatives ont fait l'objet de traitements statistiques uni- et bi-variés, en recourant notamment à des tests d'indépendance du khi-deux.

Pour compléter le recueil de données, des entretiens semi-directifs réalisés auprès de 20 diplômé.e.s ont donné lieu à une analyse de contenu thématique (Alami et al., 2019) visant à révéler les traits saillants du processus d'insertion professionnelle. Il s'agissait de rendre compte de l'architecture cognitive et affective des personnes singulières à partir des thèmes structurant l'entretien semi-directif (Bardin, 1991 [1977]; Blanchet \& Gotman, 2005 [1992]). La constitution de cet échantillon d'interviewé.e.s n'a pas donné lieu à une stratification formelle, trop contraignante au vu de la difficulté à joindre d'ancien.ne.s diplômé.e.s. L'objectif a toutefois consisté, dans la mesure du possible, à contacter un.e diplômé.e par année de sortie (de 2010 à 2014) et par type de diplôme. De la sorte, sans volonté de représentativité, les diplômé.e.s ayant été interviewé.e.s offrent un panorama varié des types et niveaux de diplômes, ainsi que de l'ancienneté de la sortie des études (voir tableau 2).

\footnotetext{
${ }^{7}$ Elles sont $49,4 \%$ parmi les répondant.e.s alors qu'elles ne sont que $47,3 \%$ dans l'échantillon initial des destinataires (différence non significative statistiquement).
} 


\section{Tableau 2}

Caractéristiques des diplômé.e.s interviewé.e.s

\begin{tabular}{|c|c|c|c|c|c|}
\hline & Identité & $\begin{array}{l}\text { Diplôme(s) } \\
\text { STAPS } \\
\end{array}$ & Autre(s) diplôme(s) & $\begin{array}{c}1^{\mathrm{er}} \text { emploi } \\
\text { (après études) }\end{array}$ & $\begin{array}{l}\text { Emploi actuel } \\
\text { (au 01/01/2016) }\end{array}$ \\
\hline 1 & $\begin{array}{l}\mathrm{F}-\mathrm{CH} \\
26 \text { ans }\end{array}$ & $\begin{array}{l}\text { Deust SIS } \\
(2014)\end{array}$ & $\begin{array}{l}\text { BAFA } \\
\text { Éducatrice spécialisée } \\
\text { (VAE) }\end{array}$ & $\begin{array}{l}\text { Coordinatrice socio- } \\
\text { éducative (CDD) }\end{array}$ & $\begin{array}{l}\text { Éducatrice spécialisée } \\
\text { (CDI) }\end{array}$ \\
\hline 2 & $\begin{array}{l}\text { F-AF1 } \\
25 \text { ans }\end{array}$ & $\begin{array}{l}\text { Deust SIS } \\
(2014)\end{array}$ & Brevet d'État ski & $\begin{array}{l}\text { Gendarme } \\
\text { Monitrice de ski (à } \\
\text { temps partiel) }\end{array}$ & $\begin{array}{l}\text { Gendarme } \\
\text { Monitrice de ski (à } \\
\text { temps partiel) }\end{array}$ \\
\hline 3 & $\begin{array}{l}\text { H-GS } \\
32 \text { ans }\end{array}$ & $\begin{array}{l}\text { Deust SPA } \\
(2010)\end{array}$ & $\begin{array}{l}\text { CAP des métiers d'art, } \\
\text { brevet des métiers d'art } \\
(2013-2016)\end{array}$ & $\begin{array}{l}\text { Éducateur sportif en } \\
\text { institut médico- } \\
\text { professionnel }\end{array}$ & $\begin{array}{l}\text { Réparateur } \\
\text { d'instrument de } \\
\text { musique }\end{array}$ \\
\hline 4 & $\begin{array}{l}\text { F-CB } \\
30 \text { ans }\end{array}$ & $\begin{array}{l}\text { Deust APSL } \\
\text { Seniors (2011) }\end{array}$ & $\begin{array}{l}\text { Licence professionnelle } \\
\text { des métiers du tourisme } \\
\text { et des loisirs sportifs } \\
\text { (2012) }\end{array}$ & $\begin{array}{l}\text { Éducatrice sportive } \\
\text { (CDD à temps partiel), }\end{array}$ & $\begin{array}{l}\text { Directrice d'une } \\
\text { association sportive } \\
\text { prestataire d'activités } \\
\text { physiques dans les } \\
\text { écoles }\end{array}$ \\
\hline 5 & $\begin{array}{l}\text { H-GK } \\
30 \text { ans }\end{array}$ & $\begin{array}{l}\text { Licence APAS } \\
\text { (2011) }\end{array}$ & $\begin{array}{l}\text { BTS Assistant de } \\
\text { gestion }(2010) \\
\text { Diplôme fédéral de } \\
\text { handball }\end{array}$ & $\begin{array}{l}\text { Assistant d'éducation } \\
\text { (CDD) }\end{array}$ & $\begin{array}{l}\text { Éducateur spécialisé } \\
\text { (CDI) }\end{array}$ \\
\hline 6 & $\begin{array}{l}\text { H-JS } \\
32 \text { ans }\end{array}$ & $\begin{array}{l}\text { Licence ENT } \\
\text { (2011) } \\
\text { Licence MS } \\
(2010)\end{array}$ & $\begin{array}{l}\text { Concours Conseiller } \\
\text { territorial des APS } \\
(2015) \\
\text { Diplômes fédéraux } \\
\text { d'entraîneur en football }\end{array}$ & $\begin{array}{l}\text { Surveillant en école } \\
\text { (CDD) et éducateur } \\
\text { sportif à temps partiel }\end{array}$ & $\begin{array}{l}\text { Responsable d'une } \\
\text { piscine dans } \\
\text { l'Eurométropole }\end{array}$ \\
\hline 7 & $\begin{array}{l}\text { F-HD } \\
27 \text { ans }\end{array}$ & $\begin{array}{l}\text { Licence MS } \\
(2013) \\
\text { L-Pro CPSS } \\
(2014)\end{array}$ & (2) & $\begin{array}{l}\text { Vendeuse dans un } \\
\text { magasin de sport } \\
\text { (CDD puis CDI) }\end{array}$ & $\begin{array}{l}\text { Co-gérante d'un } \\
\text { restaurant alsacien }\end{array}$ \\
\hline 8 & $\begin{array}{l}\text { H-AM } \\
27 \text { ans }\end{array}$ & $\begin{array}{l}\text { L-Pro CPSS } \\
(2014)\end{array}$ & $\begin{array}{l}\text { DUT Logistique (2012) } \\
\text { Licence professionnelle } \\
\text { Logistique (2013) }\end{array}$ & Logisticien & $\begin{array}{l}\text { Clerc significateur } \\
\text { d'huissier de justice }\end{array}$ \\
\hline 9 & $\begin{array}{l}\text { F-DA } \\
28 \text { ans }\end{array}$ & $\begin{array}{l}\text { L-Pro CPSS } \\
(2013)\end{array}$ & $\begin{array}{l}\text { BTS Communication } \\
(2012)\end{array}$ & $\begin{array}{l}\text { Responsable de rayon } \\
\text { Decathon (CDI) }\end{array}$ & $\begin{array}{l}\text { Militaire dans l'Armée } \\
\text { de terre (sous-officier) }\end{array}$ \\
\hline 10 & $\begin{array}{l}\mathrm{F}-\mathrm{CP} \\
28 \text { ans }\end{array}$ & $\begin{array}{l}\text { L-Pro CPSS } \\
(2013)\end{array}$ & $\begin{array}{l}\text { BTS NRC (2012) } \\
\text { Master marketing vente } \\
\text { et distribution au } \\
\text { CNAM (2016) }\end{array}$ & $\begin{array}{l}\text { Chargée administration } \\
\text { des ventes et suivi des } \\
\text { grands comptes } \\
\text { (commerce de gros de } \\
\text { produits froids) }\end{array}$ & $\begin{array}{l}\text { Chargée administration } \\
\text { des ventes et suivi des } \\
\text { grands comptes } \\
\text { (commerce de gros de } \\
\text { produits froids) }\end{array}$ \\
\hline 11 & $\begin{array}{l}\text { F-PR } \\
36 \text { ans }\end{array}$ & $\begin{array}{l}\text { DEUST SIS } \\
(2007) \\
\text { Licence APAS } \\
(2008) \\
\text { Master APAS } \\
(2010)\end{array}$ & / & $\begin{array}{l}\text { Éducatrice spécialisée } \\
\text { dans le secteur } \\
\text { médico-social (plus de } \\
10 \text { années) }\end{array}$ & $\begin{array}{l}\text { Conseillère en } \\
\text { insertion } \\
\text { professionnelle en } \\
\text { mission locale (CDI) }\end{array}$ \\
\hline 12 & $\begin{array}{l}\text { H-JC } \\
31 \text { ans }\end{array}$ & $\begin{array}{l}\text { Licence APAS } \\
(2011) \\
\text { Master APAS } \\
(2013) \\
\text { Master ENT } \\
(2015) \\
\end{array}$ & $\begin{array}{l}\text { Diplômes fédéraux } \\
\text { dans le football }\end{array}$ & Éducateur sportif & $\begin{array}{l}\text { Coordinateur des } \\
\text { activités sportives dans } \\
\text { un club }\end{array}$ \\
\hline 13 & $\begin{array}{l}\mathrm{H}-\mathrm{CR} \\
32 \text { ans }\end{array}$ & $\begin{array}{l}\text { Master ENT } \\
(2010)\end{array}$ & l & $\begin{array}{l}\text { Coordinateur des } \\
\text { activités sportives } \\
\text { (CDD) }\end{array}$ & $\begin{array}{l}\text { Conseiller technique } \\
\text { des activités physiques } \\
\text { et sportives pour une } \\
\text { collectivité }\end{array}$ \\
\hline
\end{tabular}




\begin{tabular}{|c|c|c|c|c|c|}
\hline 14 & $\begin{array}{l}\text { F-JK } \\
29 \text { ans }\end{array}$ & $\begin{array}{l}\text { Licence ENT } \\
\text { (2011) } \\
\text { Master ENT } \\
\text { (2013) }\end{array}$ & $\begin{array}{l}\text { Monitrice fédérale en } \\
\text { gymnastique }\end{array}$ & $\begin{array}{l}\text { Assistante d'éducation } \\
\text { en lycée (CDD) }\end{array}$ & $\begin{array}{l}\text { Assistante d'éducation } \\
\text { et préparatrice } \\
\text { physique dans } \\
\text { plusieurs clubs sportifs } \\
\text { (à temps partiel) }\end{array}$ \\
\hline 15 & $\begin{array}{l}\mathrm{H}-\mathrm{BC} \\
30 \text { ans }\end{array}$ & $\begin{array}{l}\text { Licence ENT } \\
\text { (2011) } \\
\text { Master ENT } \\
\text { (2013) }\end{array}$ & $\begin{array}{l}\text { Diplômes fédéraux } \\
\text { d'entraîneur de football } \\
\text { pour jeunes et seniors }\end{array}$ & $\begin{array}{l}\text { Préparateur physique à } \\
\text { temps partiel et } \\
\text { coordinateur } \\
\text { administratif et/ou } \\
\text { pédagogique du } \\
\text { championnat } \\
\text { universitaire de } \\
\text { football }\end{array}$ & $\begin{array}{l}\text { Co-gérant salarié d'une } \\
\text { société industrielle } \\
\text { spécialisée dans les } \\
\text { enceintes acoustiques ; } \\
\text { préparateur physique à } \\
\text { temps partiel pour les } \\
\text { arbitres de football }\end{array}$ \\
\hline 16 & $\begin{array}{l}\text { H-QLD } \\
29 \text { ans }\end{array}$ & $\begin{array}{l}\text { Licence ENT } \\
\text { (2012) } \\
\text { Master ENT } \\
\text { (2014) }\end{array}$ & $\begin{array}{l}\text { Diplômes fédéraux en } \\
\text { rugby (entraîneur de } \\
\text { jeunes et de seniors) }\end{array}$ & $\begin{array}{l}\text { Éducateur sportif à } \\
\text { temps partiel dans un } \\
\text { club sportif }\end{array}$ & $\begin{array}{l}\text { Éducateur sportif } \\
\text { contractuel à temps } \\
\text { complet par le multi- } \\
\text { salariat (Éducation } \\
\text { nationale, collectivité, } \\
\text { associations sportives) }\end{array}$ \\
\hline 17 & $\begin{array}{l}\text { H-JBE } \\
30 \text { ans }\end{array}$ & $\begin{array}{l}\text { Licence ENT } \\
(2011) \\
\text { Master ENT } \\
(2013)\end{array}$ & $\begin{array}{l}\text { Diplômes fédéraux } \\
\text { d'entraîneur jeunes et } \\
\text { diplôme d'État } \\
\text { d'entraineur } \\
\text { professionnel en } \\
\text { volley-ball }\end{array}$ & $\begin{array}{l}\text { Entraîneur sportif et } \\
\text { chargé de } \\
\text { développement à la } \\
\text { Ligue d'Alsace de } \\
\text { volley-ball (temps } \\
\text { partiel) }\end{array}$ & $\begin{array}{l}\text { Préparateur physique } \\
\text { et entraîneur à temps } \\
\text { partiel ; directeur d'une } \\
\text { société de consulting } \\
\text { en préparation } \\
\text { physique et } \\
\text { réathlétisation }\end{array}$ \\
\hline 18 & $\begin{array}{l}\text { F-JD } \\
30 \text { ans }\end{array}$ & $\begin{array}{l}\text { Licence MS } \\
(2010) \\
\text { Master MGS } \\
(2012) \\
\end{array}$ & $\begin{array}{l}\text { BTS Diététique, bien- } \\
\text { être et santé }\end{array}$ & $\begin{array}{l}\text { Conseiller de vente } \\
\text { chez Decathon (CDD) }\end{array}$ & $\begin{array}{l}\text { Chargée du contrôle de } \\
\text { la qualité et de } \\
\text { l'hygiène de la } \\
\text { restauration à l'INSEP }\end{array}$ \\
\hline 19 & $\begin{array}{l}\text { H-MF } \\
30 \text { ans }\end{array}$ & $\begin{array}{l}\text { Licence MS } \\
(2011) \\
\text { Master MGS } \\
(2013) \\
\end{array}$ & I & $\begin{array}{l}\text { Chef de secteur } \\
\text { (industrie des } \\
\text { boissons) }\end{array}$ & $\begin{array}{l}\text { Category manager } \\
\text { (industrie des } \\
\text { boissons) }\end{array}$ \\
\hline 20 & $\begin{array}{l}\text { H-ER } \\
30 \text { ans }\end{array}$ & $\begin{array}{l}\text { Master MGS } \\
\text { (2013) }\end{array}$ & $\begin{array}{l}\text { Diplôme de } \\
\text { comptabilité-gestion } \\
(2010) \\
\text { Licence économie- } \\
\text { gestion (2011) } \\
\text { Master 1 } 1^{\text {ere }} \text { année } \\
\text { Administration } \\
\text { économique et sociale }\end{array}$ & $\begin{array}{l}\text { Assistant commercial } \\
\text { dans une centrale } \\
\text { d'achat de matériels } \\
\text { sportifs (CDD) }\end{array}$ & $\begin{array}{l}\text { Commercial pour un } \\
\text { distributeur de matériel } \\
\text { de bureau }\end{array}$ \\
\hline \multicolumn{6}{|c|}{$\begin{array}{l}\text { SIS : sport et inadaptations sociales } \\
\text { SPA : sport adapté } \\
\text { APSL Seniors : activités physiques sportives et de loisirs pour publics seniors } \\
\text { APAS : activités physiques adaptées et santé } \\
\text { ENT : entraînement } \\
\text { MS : management du sport }\end{array}$} \\
\hline
\end{tabular}

\section{Table 2}

\section{Characteristics of the graduates interviewed}

L'étude, dans ses deux volets complémentaires, le questionnaire et l'entretien, cherche ainsi à dévoiler les perceptions, les représentations qu'ont les diplômé.e.s de la contribution de la formation et/ou des diplômes à l'accès à l'emploi et à la nature de l'emploi occupé. 


\section{Figure 1}

L'offre de formation à la Faculté des sciences du sport de Strasbourg

L'offre de formations se compose des formations suivantes :

.Deust (bac+2) :

- métiers de la forme

- sport adapté

- APS et inadaptation sociale

- activités physiques sportives et de loisirs pour les publics seniors

.Licence professionnelle (bac +3$)$ :

- commercialisation des produits et des services sportifs

.Licence STAPS $(\mathrm{bac}+3)$ mention :

- activités physiques adaptées et santé

- éducation physique

- entraînement

- management du sport

.Master STAPS (bac+5) spécialité :

- activités physiques adaptées et santé, ingénierie et développement

- entraînement sport et santé

- marketing et gestion du sport

(dont une spécialisation European resort and wellness manager)

- politiques sportives et aménagement des territoires

.Master enseignement de l'éducation physique (bac+5)

NB : Dans l'enquête ont été pris en compte les différents niveaux de diplômes puisqu'ils structurent également l'offre de formation.

\section{Figure 1}

The training offer at the Faculty of sports sciences from Strasbourg

Soulignons les modalités de fonctionnement de ces diplômes. Les Deust et la licence professionnelle, diplômes dits «professionnalisants » (visant une insertion professionnelle directe après l'obtention du diplôme), sont organisés en alternance et bénéficient de l'intervention de nombreux professionnels spécialistes des secteurs d'emplois visés. Proposées en formation initiale, les licences prévoient plusieurs périodes de stage dont un en troisième année (de 5 semaines à 4 mois). Pour leur part, les masters intègrent des stages longs (de 4 à 6 mois) en première et deuxième année permettant aux étudiant.e.s d'acquérir une expérience professionnelle significative, renforcée au cours du cursus par les interventions et l'expertise de professionnels.

En dehors du ou des diplômes possédés en STAPS, une attention particulière a aussi été portée aux autres qualifications universitaires obtenues (avant l'entrée en STAPS ou a posteriori), aux qualifications certifiées par Jeunesse et Sport (du type brevet fédéral ou d'État) et à des qualifications plus spécifiques (comme une formation en nutrition décernée par un institut privé). Parmi l'ensemble des répondant.e.s, la part des diplômé.e.s faisant état de qualifications plurielles (qu'elles soient fédérales, universitaires ou autres) s'élève à un peu moins de $40 \%$. Le profil majoritaire reste celui d'un parcours de formation spécifique en sciences du sport $(62,1 \%)$ (voir tableau 3$)$. Tenant compte de cette indication, nous avons analysé plus en détail les parcours et situations d'emploi de ces diplômé.e.s au profil de qualification différencié, ainsi 
que les effets de l'éventuelle multi-possession de diplômes et de la valence "sportive » qui caractérise ou non les emplois occupés sur la nature des ressentis (ou ressentiments).

\section{Tableau 3}

Profil des qualifications détenues $(n=446)$

\begin{tabular}{|c|c|c|}
\hline & Effectifs & $\%$ \\
\hline Une ou plusieurs qualifications en Staps & 276 & $61.9 \%$ \\
\hline $\begin{array}{l}\text { Une ou plusieurs qualifications en Staps et autres } \\
\text { qualifications de l'enseignement supérieur }\end{array}$ & 106 & $23.8 \%$ \\
\hline $\begin{array}{l}\text { Une ou plusieurs qualifications en Staps et une } \\
\text { qualification Jeunesse et Sport ou formations } \\
\text { fédérales }\end{array}$ & 58 & $13 \%$ \\
\hline $\begin{array}{l}\text { Une ou plusieurs qualifications en Staps et } \\
\text { qualification non universitaire autres que } \\
\text { Jeunesse et Sport et/ou formations fédérales }\end{array}$ & 6 & $1.3 \%$ \\
\hline Ensemble & 446 & $100 \%$ \\
\hline
\end{tabular}

Table 3

Profile of the detained qualifications $(n=446)$

\section{III - Quelques caractéristiques de la situation d'emploi des diplômé.e.s STAPS de Strasbourg}

Notre enquête ne prétend ni à la représentativité des diplômé.e.s STAPS, ni à établir des généralités, mais vise à décrire des processus sociaux à l'œuvre dans les parcours d'insertion des diplômé.e.s à une échelle locale en lien avec les spécificités du marché de l'emploi local. Pour faciliter la compréhension de nos résultats, nous mobilisons dans un premier temps les données statistiques de l'enquête quantitative afin de contextualiser les propos rapportés et présentés dans un second temps à l'aide de l'enquête qualitative par entretien.

À titre indicatif, l'insertion professionnelle dans le secteur marchand des diplômé.e.s de la filière management du sport tient pour partie à la présence locale des sièges sociaux français d'entreprises multinationales telles que Adidas, Canyon, Columbia, Puma et d'autres entreprises commercialisant des équipements sportifs comme Casal Sport. Des particularités locales liées à la fois aux caractéristiques de l'enquête, à l'offre de formation et à la structure du marché de l'emploi sont en mesure d'expliquer les différences de résultats constatés entre notre étude quantitative et celle réalisée par le GAREF ${ }^{8}$ auprès des diplômé.e.s STAPS 20152016.

\footnotetext{
${ }^{8}$ Le Groupe d'Analyse de la Relation Emploi Formation (GAREF) est une structure conventionnelle qui lie quatre entités : la Commission Paritaire Nationale Emploi, Formation (CPNEF) sport et les universités de Paris-Est Créteil, Paris-Est Marne-la-Vallée et Lyon. Le GAREF mobilise une douzaine de chercheurs qui répondent aux besoins des acteurs en matière d'analyse de l'emploi. Cette enquête a été réalisée pour la C3D (conférence des directeurs et doyens STAPS) entre mai 2018 et septembre 2018 auprès de diplômé.e.s en 2015-2016 titulaires d'un diplôme STAPS (Deust, licence 3 générale, licence professionnelle, master 2). Voir: https://c3dstaps.fr/2019/01/09/linsertion-professionnelle-des-diplomes-de-staps/\#more-1361.
} 


\section{Tableau 4}

Caractéristiques des situations d'emploi des diplômé.e.s 2010-2014 interrogé.e.s au regard des données de l'enquête du GAREF

\begin{tabular}{lcc}
\hline & $\begin{array}{c}\text { Enquête Strasbourg } \\
\mathbf{2 0 1 0 - 2 0 1 4} \\
(\mathbf{n = 4 4 6 )}\end{array}$ & $\begin{array}{c}\text { Enquête GAREF (C3D) } \\
2015-2016 \\
(\mathrm{n}=2458)\end{array}$ \\
\hline En emploi & $\mathbf{7 9 , 8} \%$ & $56,8 \%$ \\
\hline En poursuite d'études & $\mathbf{1 1 , 7} \%$ & $33,4 \%$ \\
\hline Sans emploi & $\mathbf{8 , 5} \%$ & $9,8 \%$ \\
\hline $\begin{array}{l}\text { Possession d'autres diplômes } \\
\text { (hors STAPS) }\end{array}$ & $\mathbf{3 8 , 1} \%$ & $65,5 \%$ \\
\hline
\end{tabular}

Hormis les diplômé.e.s ayant indiqué poursuivre des études

\begin{tabular}{lcc}
\hline En emploi & $\mathbf{9 0 , 4} \%$ & $84,5 \%$ \\
\hline & Parmi les diplômé.e.s en emploi & \\
\hline CDI & $\mathbf{6 8 , 5} \%$ & $56,8 \%$ \\
\hline Temps plein & $\mathbf{8 9} \%$ & $82,8 \%$ \\
\hline Secteur privé associatif & $\mathbf{2 1 , 5 \%}$ & $22,9 \%$ \\
\hline Secteur privé marchand & $\mathbf{3 8 , 1 \%}$ & $31 \%$ \\
\hline Secteur public & $\mathbf{4 0 , 4 \%}$ & $46,1 \%$ \\
\hline Emploi hors secteur sport et loisir & $\mathbf{2 0 , 6} \%$ & $22,1 \%$ \\
\hline
\end{tabular}

\section{Table 4}

Employment situations of the 2010-2014 graduates surveyed in comparison with the GAREF survey data

Le taux d'insertion supérieur relevé à Strasbourg, ainsi que les conditions d'emploi plus favorables (en termes de type de contrat ou de quotité de travail) tiennent à la conception même de l'enquête qui porte sur une période plus éloignée de la sortie des études, comme l'attestent par ailleurs d'autres études d'insertion (MESRI, 2019). S'agissant de la répartition par secteur d'emploi, la comparaison des données montre une forte similarité entre la situation strasbourgeoise et la situation nationale.

De manière plus précise, notre enquête fait état de 54,5\% des diplômé.e.s en emploi qui ont un statut d'employé, 33,5\% de cadre, $7,7 \%$ de profession intermédiaire et $4,3 \%$ de profession libérale. Les principales professions déclarées sont celles d'éducateur.rice $(18,5 \%)$, de professeur.e d'EPS (16,9\%), de commercial.e (14,3\%) et de cadre commercial.e $(6,7 \%)$. Ces 
professions correspondent aux principaux débouchés professionnels des filières de formation de la faculté des sciences du sport de Strasbourg.

\section{a) L'accès au premier emploi, une épreuve ?}

Le premier emploi fait l'objet de luttes et d'ajustements pour être décroché ${ }^{9}$. Son accès est ainsi déclaré difficile par environ un tiers des répondant.e.s parmi lesquel.le.s $80 \%$ n'ont pas « converti » leur stage de fin d'études en emploi. Près de $35 \%$ des diplômé.e.s affirment toutefois l'avoir obtenu dans la continuité du stage de fin d'études. En recherche d'emploi pendant 10 mois après la fin de son stage long (6 mois) de fin d'étude de master, ce diplômé affirme sans détour : " j'ai eu des difficultés à obtenir des entretiens après mon master et mon stage au service des sports de la ville (...) j'ai eu l'opportunité d'être assistant commercial [CDD 6 mois renouvelé] dans une centrale d'achat de matériels sportifs d'une fédération sportive » [E.R., commercial, diplômé d'une licence économie-gestion, d'un master $1{ }^{\text {ère }}$ année administration économique et sociale, puis du master marketing et gestion du sport en 2013].

\section{Tableau 5}

Caractéristique du premier emploi occupé pour les diplômé.e.s interrogé.e.s

\begin{tabular}{lc}
\hline & \% de diplômé.e.s \\
\hline L'emploi actuel est le premier emploi & $50,4 \%$ \\
\hline CDD, contrat aidé ou intérimaire (premier emploi) & $63,7 \%$ \\
\hline Statut d'employé.e (premier emploi) & $81,7 \%$ \\
\hline Premier emploi obtenu suite à un stage & $34,6 \%$ \\
\hline Difficultés ressenties d'accéder au premier emploi & $31,9 \%$ \\
\hline avec un sentiment de difficulté liée au peu d'offres d'emploi & $28,3 \% *$ \\
\hline avec un sentiment de difficulté liée au manque de reconnaissance du diplôme & $15,0 \%$ \\
\hline Aucun lien perçu entre premier emploi et sport & $25,5 \%$ \\
\hline
\end{tabular}

*Lecture : 28,3\% des diplômé.e.s intérrogé.e.s déclarant des difficultés d'accès au premier emploi invoquent le peu d'offres d'emploi.

\section{Table 5}

First job characteristic of the graduates surveyed

Les diplômé.e.s ayant éprouvé des difficultés à accéder à leur premier emploi s'avèrent être majoritairement des personnes qui actuellement occupent un emploi de cadre, parfois encore en contrat à durée déterminée (30\%) notamment dans la fonction commerciale. Sans lien avec le sport, leur premier emploi était à durée déterminée et à temps partiel. Parmi ces diplômé.e.s, plusieurs affirment avoir fait évoluer la définition de leur projet professionnel en cours d'études et estiment aussi avoir été pénalisé.e.s par le manque de reconnaissance de leur(s) titre(s) scolaire(s) :

\footnotetext{
${ }^{9}$ Le délai d'accès au premier emploi est supérieur à 6 mois pour 38,9\% des 131 répondant.e.s.
} 
« Je suis satisfaite de mon travail même s'il ne correspond ni au niveau d'études, ni à la filière de formation. Je vais passer le diplôme d'éducateur spécialisé en VAE pour obtenir la reconnaissance qui me manque » [S.K., éducatrice spécialisée titulaire d'un Deust sport et intervention sociale obtenu en 2010].

Plus d'un.e diplômé.e sur deux (57,5\%) revoit son projet professionnel au cours de son parcours de formation. Les métiers les plus ciblés en début de formation sont par ordre de priorité celui d'animateur.rice ou d'éducateur.rice sportif.ive $(29,9 \%)$, celui de professeur.e d'EPS (24,2 \%) et les métiers en lien avec le commerce ou le marketing (19,9\%). Et seuls $6,3 \%$ des répondant.e.s déclarent être entré.e.s en formation sans projet professionnel clairement formalisé.

\section{b) De la nature de l'emploi selon les diplômes obtenus}

Nous avons aussi relevé la perception qu'ont les diplômé.e.s en situation d'emploi de la nature de ce dernier et en particulier de son caractère sportif ou non. Ainsi, près de 8 diplômé.e.s sur 10 en emploi estiment que leur emploi est en lien avec le sport. Les diplômé.e.s paraissent définir leur emploi comme étant lié au sport dès lors qu'ils exercent une profession « sportive $»^{10}$ et/ou qu'ils développent une activité qui nécessite des compétences d'encadrement sportif par exemple, ou encore lorsqu'ils travaillent dans une structure qualifiée de « sportive », c'est-à-dire dont l'activité économique est étroitement liée à l'univers sportif, aux biens ou services sportifs, même si l'emploi mobilise d'autres compétences (comme dans l'industrie du sport où prévalent des compétences de gestion, commerciales et/ou de marketing).

Les diplômé.e.s possédant une ou plusieurs qualifications exclusivement en STAPS, ainsi que celles et ceux ayant obtenu une qualification sportive (Jeunesse et Sport et/ou diplômes sportifs fédéraux) occupent plus souvent un emploi caractérisé par des situations de face à face pédagogique nécessitant des compétences d'encadrement de divers publics. Ainsi la multipossession de diplômes STAPS et « sportifs » favorise nettement l'emploi dans le domaine de l'encadrement sportif et en particulier sous le statut de profession libérale. Les multidiplômé.e.s en STAPS possédant une qualification non universitaire autre que Jeunesse et Sport et/ou formations fédérales ainsi que les multi-diplômé.e.s en STAPS et d'autres établissements de l'enseignement supérieur sont respectivement $83,3 \%$ et 33,7 \% à occuper un emploi sans aucun lien avec le sport, comparativement aux diplômé.e.s STAPS uniquement $(15,1 \%)$ ou aux diplômé.e.s STAPS possédant également une qualification Jeunesse et Sport ou fédérale $(13,1 \%)\left(\mathrm{Khi}^{2}(3)=28,93, \mathrm{p}<0,01\right)$. Plusieurs diplômé.e.s expriment leur frustration lorsque l'emploi occupé n'est pas en lien avec leur domaine et leur niveau de formation :

"Je travaillais avec des salariés qui avaient un BTS et je faisais le même boulot qu'eux, j'étais quand même sous-exploité (...) aujourd'hui, je suis commercial pour Lyreco, j'espère revenir dans le sport le plus vite possible » [E.R., op. cit., cf. supra].

Pour autant, il est des situations d'emploi sans lien avec le sport, bien vécues :

\footnotetext{
${ }^{10}$ En référence à la nomenclature de la PCS-ESE (professions et catégories socioprofessionnelles des emplois salariés des employeurs publics et privés) de 2017, il est possible de relever des professions dites « sportives » au sein de la principale rubrique 424a « Moniteurs et éducateurs sportifs, sportifs professionnels » : moniteur sportif, éducateur sportif, entraîneur sportif, sportif professionnel, guide de montagne, instructeur. Deux autres professions identifiées dans la nomenclature intègrent dans leur dénomination les activités physiques et sportives : « Conseiller des activités physiques et sportives” (333f) et « Opérateur des activités physiques et sportives » (524c).
} 
"Je suis responsable de secteur en Alsace [chez Innocent Drink] ; je gère une petite équipe de commerciaux, j'ai une voiture ; on me donne des objectifs, je les atteints " [M.F., diplômé du master marketing et gestion du sport en 2013].

\section{c) De l'effet du sexe et du niveau de diplôme}

Des différences d'emploi se font jour selon le sexe. En effet, les hommes s'avèrent plus fréquemment être en emploi ( $86,3 \%$ contre $79,1 \%$ pour les femmes), notamment dans le secteur marchand $\left(48,9 \%\right.$ vs $\left.28,1 \% ; \mathrm{Khi}^{2}(2)=16,78, \mathrm{p}<0,01\right)$ avec un statut de cadre $\left(33,7 \%\right.$ vs $\left.17,8 \% ; \mathrm{Khi}^{2}(3)=9,95, \mathrm{p}<0,05\right)$ et dans le domaine de l'entraînement sportif $\left(3,3 \%\right.$ vs $\left.0,6 \% ; \mathrm{Khi}^{2}(7)=29,61, \mathrm{p}<0,01\right)$. Les femmes sont plus nombreuses à être titulaires d'un Deust $\left(43,2 \%\right.$ vs $13,3 \%$ des hommes; $\left.\mathrm{Khi}^{2}(2)=46,81, \mathrm{p}<0,01\right)$, à occuper des emplois de professeures de fitness $\left(5,5 \%\right.$ vs $\left.1,1 \% ; \mathrm{Khi}^{2}(32)=48,26, \mathrm{p}<0,05\right)$ et à s'être insérées dans le secteur associatif $\left(28,1 \%\right.$ vs $\left.15,7 \% ; \mathrm{Khi}^{2}(2)=16,78, \mathrm{p}<0,01\right)$. Des inégalités sociales d'accès et de rentabilisation des diplômes obtenus confirment d'autres travaux soulignant le maintien voire le renforcement des divisions sexuelles de la formation STAPS et du travail sportif (Érard \& Louveau, 2016; Érard \& Guégnard, 2018) avec davantage d'hommes notamment sur les tâches spécifiques d'entraînement (Céreq, 2018). Parmi ceux.elles qui exercent leur emploi actuel dans le domaine de l'entraînement, 85,7 \% sont des hommes.

Cet état de fait corrobore l'existence d'inégalités entre hommes et femmes face à l'emploi. Pourtant, le taux d'emploi des hommes supérieur à celui des femmes n'est pas lié à la multipossession de diplômes pour laquelle n'apparait pas de différence significative. Il semblerait que des résistances mentales perdurent : les représentations sur les attributions des métiers et les capacités individuelles dépendantes du sexe pèsent toujours sur le recrutement des jeunes diplômé.e.s. (Louveau, 2013 ; Chimot, 2017).

"J'envisage de passer le concours de professeur des écoles pour obtenir de la stabilité (...) j'adore la préparation physique, mais c'est vraiment une niche, c'est très difficile d'en vivre » [J.K., assistante d'éducation en emploi principal, préparatrice physique dans un club de football féminin, diplômée d'un master spécialité entraînement en 2013].

En France, le métier d'éducateur.rice sportif.ive demeure majoritairement exercé par les hommes et si la part des femmes - $33 \%$ - a certes augmenté depuis les années 1990 (27\%), sa progression reste timide (Céreq, 2018, p. 62). À la différence des données nationales, notre enquête $^{11}$ met en évidence que les femmes sont plus nombreuses $(66,3 \%)$ que les hommes dans la filière APAS (Activités Physiques Adaptées et Santé), expliquant sans doute que parmi les diplômé.e.s qui exercent le métier d'éducateur.rice, 54,5 \% sont des femmes. Quant au métier de commercial.e exercé par $21,1 \%$ des diplômé.e.s en emploi (soit 75 individus), il semble toujours principalement " réservé » aux hommes (55 contre 20 femmes) dans un secteur (le commerce des articles de sport et de loisir) où les effectifs salariés en 2015 restent majoritairement masculins (57\%) (Forco, 2016).

Aux différenciations relatives au sexe s'ajoutent des disparités en termes de niveau de qualification. S'il semble protéger du chômage puisque les diplômé.e.s de master sont les plus nombreux.ses à être en emploi (87,1\%), des variations s'observent toutefois selon la spécialité du diplôme. Les diplômé.e.s d'un master en APAS semblent ainsi éprouver plus de difficultés

\footnotetext{
${ }^{11}$ Les différences enregistrées entre le niveau national et le niveau local peuvent s'expliquer d'une part par l'offre de formations au sein de la faculté des sciences du sport marquée par 5 diplômes du secteur APAS (Activités Physiques Adaptées et Santé) qui attirent davantage de femmes et, d'autre part par le périmètre sectoriel pris en compte dans l'étude du Céreq (2018) qui n'inclut pas le champ des secteurs social et médico-social.
} 
puisqu'ils.elles ne sont que 75,7 \% en emploi (et 18,9\% sans emploi). Les secteurs associatifs ou du médico-social sont peut-être moins pourvoyeurs d'emplois à ce niveau de qualification ${ }^{12}$ que ceux du commerce ou de l'enseignement de l'EPS :

«Je suis surqualifiée pour ce poste qui n'est pas en adéquation avec le master. J'ai obtenu cet emploi grâce à mes expériences professionnelles cumulées en parallèle de mes études. J'ai construit mon parcours pendant des années... dans l'animation, le handicap, les maisons de retraite, à l'hôpital. Sans ces expériences, le master ne vaut rien » [A. F., chargée de développement, titulaire d'un master APAS en 2010].

\section{IV - Les liens formation-emploi « brouillés » par des situations d'emploi « bricolées »?}

Les chiffres d'insertion professionnelle plutôt positifs d'un point de vue général masquent des disparités de situations d'emploi. Loin d'être uniformes, ces dernières reflètent la réalité du marché de l'emploi sportif où apparaissent une forme d'emprise des diplômes (Dubet et al., 2010) et certaines inégalités sociales.

\section{a) Des pas de côté subis et voulus}

Il apparaît hasardeux d'identifier des emplois types en correspondance avec un diplôme donné. Les éducateurs.rices sportif.ive.s peuvent être titulaires d'un Deust, d'une licence et/ou d'un master. Et, pour celles et ceux qui sont titulaires d'une licence, et plus encore d'un master, s'exprime parfois un sentiment de déclassement :

"J'ai dû m'éloigner de la compétition sportive et de la performance; ça manquait de mises en relation avec les structures de haut niveau; aujourd'hui je suis éducateur sportif intervenant en associations et professeur d'EPS à temps partiel » [Q.L.D., diplômée du master spécialité entraînement en 2014].

La stabilité recherchée dans une situation professionnelle peut s'exprimer dans deux projets coconstruits parallèlement sans lien entre eux et aboutis. Le parcours professionnel de ce diplômé du master Marketing et gestion du sport (2012) au terme d'un cursus STAPS révèle des agencements d'activités atypiques, surprenants mais réfléchis. En 2012, il poursuit son activité de vendeur à temps partiel dans une boutique de la marque de sport ASICS, tout en créant, avec deux associés, une entreprise prestataire de restauration rapide qui va assez rapidement péricliter faute d'un investissement en temps suffisant. Après avoir quitté son emploi de vendeur, le diplômé se lance dans une carrière de mannequin l'amenant à voyager en Europe et en Asie durant trois années. De retour en France, il devient éducateur sportif contractuel dans une collectivité, avant de décrocher un CDI suite à l'obtention du concours d'éducateur territorial des APS. « Entrepreneur dans l'âme », il se lance parallèlement, avec un associé, dans la création d'une nouvelle entreprise prestataire de location d'appartements touristiques : «je m'épanouis en tant qu'éducateur sportif, j'interviens dans divers quartiers, auprès de jeunes et de seniors, je propose du fitness, de la marche nordique (...) à côté, j'ai créé ma société de location d'appartements de tourisme, on a aujourd'hui 40 appartements en location, que les propriétaires nous donnent en location ; je suis actionnaire de la société (...) pour la création de ma société, le master m'a servi, le marketing, la communication, les études ». Si a priori

\footnotetext{
${ }^{12}$ D'après les chiffres clés publiés en septembre 2010 dans le guide des activités et des métiers du secteur social, médico-social et petite enfance, la part des directeurs, cadres sociaux et éducatifs dans les professions sociales ne représente que $3 \%$ des 900000 emplois. Voir: https://solidarites-sante.gouv.fr/IMG/pdf/guide_activites et metiers du secteur social medico social et petite enfance sept 2010.pdf.
} 
l'occupation d'un poste de technicien au regard de la possession d'un diplôme de niveau master peut être jugée déqualifiante, le diplômé affirme avoir choisi sa situation professionnelle et valorise son diplôme par la cogestion de son entreprise. En creux de son double investissement dans des mondes professionnels différents, le statut de fonctionnaire pour son emploi principal lui apporte l'exercice d'un métier le satisfaisant et une situation stable, lui permettant de s'engager dans une activité d'entrepreneuriat plus risquée.

L'adéquation improbable entre type de formation et type d'emploi relève également des évolutions des objectifs professionnels en cours de formation comme en témoigne l'expérience ci-après qui met en lumière des chemins empruntés perturbés et parasités :

«Je regrette de ne pas avoir accepté la proposition d'embauche à la suite de mon alternance chez Horizon Vertical ; mais c'était compliqué, tout le monde, mes parents, mes amis, me disaient continue tes études, il faut faire un master (...) j'aimerais travailler dans le domaine du sport et dans le commerce du sport » [C.P., chargée d'administration des ventes dans une entreprise de commerce de gros dans le froid et la climatisation, titulaire d'un BTS NRC, puis d'une licence professionnelle « commercialisation des produits et des services sportifs » en 2013, puis d'un master marketing vente $\&$ distribution - CNAM].

\section{b) La multi-possession de diplômes : rempart ou ressource?}

Nous nous interrogeons sur le lien entre l'emploi occupé et la multi-possession de diplômes et ce, au regard des principaux métiers exercés - éducateur.rice, professeur.e d'EPS, commercial.e et cadre commercial.e. Les éducateurs.rices sont environ $15 \%$ à détenir, en plus de leur(s) diplôme(s) en STAPS, une qualification Jeunesse et Sport ou fédérale. Ces éducateurs.rices, relativement jeunes ( $42,4 \%$ ont entre 20 et 24 ans), occupent plus souvent des emplois précaires (notamment à temps partiel pour $16,7 \%$ vs moins de $5 \%$ pour chacune des trois autres principales professions ; $\left.\mathrm{Khi}^{2}(3)=15,56, \mathrm{p}<0,01\right)$ expliquant, selon nous, leur insatisfaction plus prononcée par rapport à leur emploi et leur sentiment d'occuper un emploi en inadéquation avec leur niveau d'études. À l'inverse, les professeur.e.s d'EPS s'estiment satisfait.e.s de leur emploi à 93,3\%, tout comme il.elle.s pensent que leur emploi est en adéquation avec leur niveau d'études $(91,7 \%)$ et leur domaine de formation $(86,7 \%)$. Il.elle.s se distinguent aussi par leur possession de plusieurs qualifications et notamment la combinaison de diplômes en STAPS et de qualifications sportives (fédérale ou Jeunesse et Sport) (23,3\% comparativement à $15,2 \%$ pour les éducateurs.rices, $7,8 \%$ pour les commerciaux.ales et $4,2 \%$ pour les cadres commerciaux.ales ; $\left.\operatorname{Khi}^{2}(9)=40,8, \mathrm{p}<0,01\right)$.

Les commerciaux.ales et cadres commerciaux.ales attestent plus souvent de parcours «nonlinéaires » qui combinent plusieurs types de qualification (universitaire hors STAPS et/ou sportive par exemple). La double qualification universitaire, qu'elle soit antérieure ou postérieure à l'obtention du ou des diplômes en STAPS, caractérise les diplômé.e.s occupant ces emplois de commerciaux.ales $(39,2 \%$ ont cette double qualification) ou cadres commerciaux.ales $(45,8 \%)$. Il s'agit plus souvent d'hommes exerçant un métier éloigné du monde de la pratique sportive. Les commerciaux.ales, au contraire des cadres commerciaux.ales, expriment l'inadéquation de leur niveau d'études et de leur domaine de formation par rapport à l'emploi occupé au moment de l'enquête.

"J'espérais autre chose que de devenir vendeuse [dans un magasin Decathlon] à la sortie de mon master » [J.D., aujourd'hui reconvertie en tant que diététicienne à l'INSEP, après 3 années d'expérience dans la vente, diplômée d'un master marketing et gestion du sport en 2012]. 
Si un certain nombre des titulaires d'un master (58,8\%) ressentent leur situation actuelle comme une forme de déclassement (Giret et al., 2006), elle peut n'être que temporaire et liée au contexte de chômage structurel modifiant la régulation diplôme-emploi.

Ces principaux profils professionnels expriment des points de vue contrastés quant à l'utilité de la multi-possession de diplômes. Ainsi, pour les répondant.e.s en emploi au moment de l'enquête, 53,9\% estiment que la possession de plusieurs diplômes (universitaires et/ou sportifs) favorise l'insertion professionnelle. 11,5\% des diplômé.e.s en emploi estiment même que la possession de plusieurs diplômes est une condition indispensable pour trouver un emploi, avis défendu notamment par les diplômé.e.s titulaires d'une qualification sportive (Jeunesse et sport ou fédérale) et du master en spécialité entraînement.

"Le master ne sert à rien si on n'a pas le brevet d'État dans sa discipline » [J.B.E., entraîneur sportif et chargé de développement à la Ligue d'Alsace de volley-ball, diplômé d'un master spécialité entraînement en 2013].

Un peu plus d'un tiers des diplômé.e.s en emploi (34,6 \%) considèrent quant à eux que cette multi-possession de diplômes n'est pas un facteur déterminant de l'accès à l'emploi. Il s'agit plus souvent de diplômé.e.s plus âgé.e.s (30-34 ans) avec une ou plusieurs qualifications en STAPS uniquement (39,9 \% vs 34,6 \% de l'ensemble des diplômé.e.s en emploi estimant que ce n'est pas un facteur déterminant), un premier emploi sans lien avec le sport (48,7\%) et plus rarement obtenu à la suite du stage de fin d'études $(44,1 \%)$.

\section{c) Des situations d'emploi « bricolées » et « resocialisées »}

La précarité de l'emploi est accrue dans certains secteurs moins favorables que d'autres à la stabilisation des situations professionnelles, comme le secteur associatif. Cette précarité s'atteste par la pluriactivité structurelle observée qui engendre des situations professionnelles chaotiques atypiques où se combinent plusieurs emplois à temps partiel.

"Je cumule plusieurs emplois : je suis gérant salarié d'une entreprise spécialisée dans la fabrication d'enceintes acoustiques, je sais, ça n'a rien à voir avec le sport, mais bon, il faut manger... À côté, je suis préparateur physique pour les arbitres de la ligue d'Alsace comme vacataire, je suis aussi responsable de l'option foot à l'École de management, je suis coordinateur du championnat de football universitaire (...) j'aimerais trouver un job fixe, mais pour être préparateur physique, il faut du bon réseau, ça fait la différence » [B.C., diplômé d'un master entraînement sportif en 2013, titulaire de diplômes fédéraux en football].

Observant la rupture nette avec une conception « tubulaire » de l'enseignement supérieur selon laquelle chaque filière constitue une espèce de couloir étanche aligné à côté des autres (Bodin \& Orange, 2013) et admettant donc la circulation des étudiant.e.s au sein de filières de formation connexes, comment pouvons-nous interpréter ces parcours de formation non linéaires et hybrides?

Au début de leurs études, les diplômé.e.s n'ont pas nécessairement clairement identifié les étapes de formation favorisant la réussite de leur projet professionnel. Les parcours sinueux, entrecoupés de reprise d'études, observés après l'obtention de leur dernier diplôme, laissent à penser des projets professionnels initiaux peu matures lors de l'entrée en formation. À cela s'ajoute un marché de l'emploi qui rend incertaine la définition assurée d'un projet professionnel. Ainsi, déclarer vouloir travailler dans le commerce et dans le sport «parce que ce sont des domaines qui [me] plaisent ", n'exonère pas de possibles réorientations professionnelles. 
"Je ne prenais pas vraiment mon pied dans une boutique de sport (...) j'ai eu l'opportunité de prendre la gérance d'un restaurant avec mon copain » [H.D., diplômée de la licence management du sport en 2013, de la licence professionnelle commercialisation des produits et des services sportifs en 2014].

Cette autre diplômée en 2014 de cette même licence professionnelle, après un BTS Communication, ex-judokate sportive de haut niveau, décide de s'engager dans l'armée et l'école des sous-officiers, "son rêve de gosse ». Pourtant, une carrière toute tracée lui était offerte chez Decathlon, là où elle fait son apprentissage et est recrutée sur un poste de responsable de rayon à la sortie de ses études; mais "le commerce, c'est un peu pesant au fil du temps », elle préfère "la rigueur, l'ordre (...) des choses que je retrouve [à l'Armée] que j'avais dans ma pratique du judo ».

Ou encore cet étudiant titulaire d'un DUT et d'une licence professionnelle en logistique, qui se réoriente vers une formation de greffier, suivant par-là les traces de son père huissier de justice, après avoir obtenu la licence professionnelle commercialisation des produits et des services sportifs en 2014 :

«Je me suis rendu compte que je n'avais pas suffisamment de contacts dans le secteur du sport, c'est un petit monde à part (...) J'ai choisi la facilité et la sécurité grâce aux connaissances de mes parents dans le secteur juridique ».

La forte incertitude sur l'emploi ne prédispose pas à la planification des parcours. Ce que l'on nomme comme étant des effets d'opportunités professionnelles résulte de situations de mise en réseaux professionnels (" après 10 mois de recherche, j'ai trouvé mon premier job grâce à une relation personnelle d'un de mes profs ») et révèle l'importance de l'immersion précoce des étudiant.e.s bien avant leur entrée dans l'enseignement supérieur et au cours de leurs études dans les divers mondes sociaux et de travail spécifiques, définis par des fonctionnements indigènes et des réseaux de relations propres (" je suis devenu co-gérant salarié de l'entreprise d'un de mes oncles »). Notre recherche confirme l'importance des « liens faibles » pour faciliter l'accès au monde du travail (Amarillo \& Mossé, 2010) :

" J'ai accumulé tous les diplômes fédéraux et d'État possibles en volley, j'ai obtenu le master [spécialité entraînement sportif], en fait j'ai une triple casquette, je suis salarié de mon club semi-professionnel à temps partiel, je suis embauché par la ligue régionale à mi-temps et j'exerce en indépendant en tant que préparateur physique, j'interviens au pôle espoirs pour les sportifs de haut niveau » [J.B.E., diplômé de master spécialité entraînement en 2013].

Ce parcours de formation témoigne d'une situation d'emploi non pas subie, mais réfléchie et arrangée en fonction des ressources individuelles du diplômé et des contraintes caractérisant les employeurs associatifs du secteur du sport. Pour saisir les parcours des diplômé.e.s, il est nécessaire de penser ensemble les contextes sociétaux, les secteurs professionnels, les normes qui s'y développent et les choix individuels, afin de mieux comprendre leurs agencements (Becquet \& Bidart, 2013).

Ainsi, la construction biographique des capacités d'adaptation aux situations et aux conjonctures sectorielles et/ou politiques des diplômé.e.s est un critère décisif de leur insertion sur le marché du travail, celui qui est régi par la logique sélective des « compétences » (Rose, 1998) et des capacités perçues, supposées et/ou réelles. 


\section{Conclusion}

Les témoignages montrent la force d'une croyance dominante en France: le destin professionnel des diplômé.e.s dépend de leurs titres scolaires. Les propos et jugements délivrés par les diplômé.e.s attestent d'un rapport instrumental aux diplômes qui constituent un moyen de reconnaissance pour accéder à ou occuper un emploi et un repère par rapport auquel les diplômé.e.s donnent une cohérence à leur parcours. Ainsi, s'agissant des diplômé.e.s STAPS, la multi-possession de qualifications sportives renforce les chances d'occuper un emploi dans l'encadrement sportif caractérisé par des situations de face à face pédagogique nécessitant des compétences de gestion de publics spécifiques. Les multi-diplômé.e.s STAPS et autres qualifications de l'enseignement supérieur occupent le plus souvent un emploi de cadre et exercent aussi hors du champ sportif. La multi-possession de diplômes ne protège pas des inégalités entre les sexes qui perdurent face à l'emploi. Les champs professionnels sont en effet marqués historiquement par des tendances genrées qui persistent : si la fonction commerciale est fortement prisée par les diplômés, la profession d'éducateur.rice sportif.ive est largement plus féminisée et exercée dans le secteur associatif.

Mais la manière dont il.elle.s analysent a posteriori leur insertion professionnelle et leur situation d'emploi est brouillée. Les parcours de formation font apparaître des variations liées à la possession de plusieurs diplômes, aux expériences diverses de pré-professionnalisation, aux reprises d'études, aux changements d'orientation de formation. Bien que l'appréciation et l'argumentation soient liées à l'emprise du diplôme, ce dernier ne peut être tenu pour seul élément explicatif. Des engagements pluriels des diplômé.e.s ont des incidences sur leur appréciation de leur retour sur investissement relatif à l'emploi occupé et au début de parcours professionnel. Il conviendrait aussi de prendre en compte les divers engagements et les investissements psychologiques et temporels réalisés par les diplômé.e.s dans les sphères d'activités connexes (secteur des loisirs, activité bénévole, missions professionnelles occasionnelles, etc.) constitutifs de dynamiques de parcours sociaux (Caradec et al., 2012) en vue d'expliquer et de comprendre leurs effets sur les situations et les dynamiques d'emplois. Les parcours de formation non linéaires et hybrides observés attestent de situations d'emploi bricolées selon les ressources individuelles des diplômé.e.s, les espaces de socialisation réactivés et les contraintes structurelles propres aux secteurs professionnels du sport.

Aussi une démarche holistique prenant en compte les divers domaines de la vie sociale et leurs connexions permettrait de mieux comprendre l'articulation entre le parcours de formation et l'emploi à partir de la manière dont les diplômé.e.s vivent leurs difficultés d'accès à l'emploi, du sens qu'ils donnent à leurs expériences et à leur insertion dans la vie professionnelle et plus fondamentalement à « ce que travailler veut dire » (Zimmermann, 2011).

Le relevé de caractéristiques individuelles relatives au parcours de formation et à l'emploi occupé ne doit pas occulter le complexe d'acteur.rice.s, de dispositifs et d'institutions participant au recrutement, à l'intermédiation vers l'emploi, au tri dont sont l'objet les diplômé.e.s (Rose, 1998). Car c'est moins le diplôme atteint qui semble déterminer l'accès à l'emploi et l'employabilité que la pertinence des reformations (Pollien, 2010) appréciée par l'ensemble des acteur.rice.s impliqué.e.s dans le recrutement (les diplômé.e.s, les employeurs et d'autres intermédiaires). 


\section{Références bibliographiques}

Alami, S., Desjeux, D., \& Garabuau-Moussaoui, I. (2019). Les méthodes qualitatives (3 ${ }^{\mathrm{e}}$ ed.). Presses Universitaires de France.

Amarillo, H., \& Mossé Ph. (2010). Heurts et malheurs des "liens forts" : l'intermédiation de l'emploi dans le secteur sport. In L. Lima \& Ph. Mossé (Eds.), Le sport comme métier ? Les STAPS des études à l'emploi (pp.117-134). Octarès.

Arliaud, M., \& Eckert, H. (dir.) (2002). Quand les jeunes entrent dans l'emploi. La Dispute.

Bardin, L. (1991). L'analyse de contenu (6 $6^{\mathrm{e}}$ ed.). Presses Universitaires de France.

Becquet, V., \& Bidart, C. (2013). Introduction. Parcours de vie, réorientations et évolutions des normes sociales. Agora débats/jeunesses, 65, 51-60. https://doi.org/10.3917/agora.065.0051

Blanchet, A., \& Gotman, A. (2005) [1992]. L'enquête et ses méthodes. L'entretien (6 $\left.{ }^{\mathrm{e}} \mathrm{ed}.\right)$. Armand Colin.

Bodin, R., \& Orange, S. (2013). L'Université n'est pas en crise. Les transformations de l'Enseignement supérieur : enjeux et idées reçues. Éditions du Croquant.

Cahuzac, É., \& Giret, J. (2001). Quand la vie professionnelle commence avant la fin des études : l'insertion des étudiants français. Reflets et perspective de la vie économique, 1-2, 37-49. https://doi.org/10.3917/rpve.401.0037

Caradec, V., Ertul, S., \& Melchior, J-Ph. (Eds.) (2012). Les dynamiques des parcours sociaux. Temps, territoires, professions. Presses Universitaires de Rennes.

Céreq Enquêtes (2018). Regards croisés sur le secteur des activités sportives et le métier d'éducateur sportif. Portrait statistique Emploi Formation, 2, co-édition Céreq et Direction des Sports du ministère des Sports.

Chimot, C. (2017). Femmes dirigeantes et cadres des organisations sportives : la construction des inégalités de carrière. Jurisport, 171, 18-21.

Dubar, C. (1999). Réflexions sociologiques sur la notion d'insertion. In B. Charlot \& D. Glasman (Eds.). Les jeunes, l'insertion, l'emploi (pp. 2-37). Presses Universitaires de France.

Dubar, C. (2001). La construction sociale de l'insertion professionnelle. Éducation et sociétés, 7, 23-36. https://doi.org/10.3917/es.007.0023

Dubet, F., Duru-Bellat, M. \& Vérétout, A. (2010). Les sociétés et leur école. Emprise du diplôme et cohésion sociale. Seuil.

Érard, C., \& Guégnard, C. (2018). (In)fortunes professionnelles des femmes à la sortie d'une filière universitaire masculine, les STAPS. Formation emploi, 2, 79-98. https://doi.org/10.4000/formationemploi.5879

Érard, C., \& Louveau, C. (2016). Entre transgression et reproduction des normes de genre. Les effets paradoxaux du goût du sport sur l'orientation scolaire et professionnelle des normaliennes en sciences du sport et éducation physique. Sciences sociales et sport, 9, 83-113. https://doi.org/10.3917/rsss.009.0083

Ertul, S., Melchior, J.-Ph., \& Lalive d'Epinay C. (Eds.) (2014). Subjectivation et redéfinition identitaire. Presses Universitaires de Rennes.

Gautié, J. (1993). Les politiques de l'emploi. Les marges étroites de la lutte contre le chômage. Vuibert. 
Giret, J-F., Nauze-Fichet, E., \& Tomasini, M. (2006). Le déclassement des jeunes sur le marché du travail. Données sociales. La société française (pp.306-314). INSEE.

Leroux, N., Haschar-Noé, N., Le Roux, N., \& Gojard, L. (2010). Rapport au travail et rapport à l'emploi des sortants de la filière STAPS. In L. Lima \& Ph. Mossé (Eds.), Le sport comme métier? Les STAPS des études à l'emploi (pp. 83-100). Octarès.

Lima, L., \& Mossé, P. (Eds.) (2010). Le sport comme métier? Les STAPS des études à l'emploi. Octarès.

Louveau, C. (2013). Les femmes dans le sport : inégalités et discriminations. In B. Andrieu (éd.), L'éthique du sport (pp. 475-489). Âge d'homme.

Mahlaoui, S. (Eds.) (2016). L'articulation des démarches quantitative et qualitative pour analyser le travail : mythe ou réalité ? Relief, 52, 92p. https://www.cereq.fr/larticulation-desdemarches-quantitative-et-qualitative-pour-analyser-le-travail-mythe-ou-realite

Mériaux, O., \& Verdier, É. (2009). Gouvernances territoriales et émergence d'une politique du rapport salarial. Espace et Sociétés, 136-137, 17-31. https://doi.org/10.3917/esp.136.0017

Ministère de 1'Enseignement supérieur, de la Recherche et de l'Innovation (2019), L'état de l'Enseignement supérieur, de la Recherche et de l'Innovation en France, 12, 140p.

Nicole-Drancourt, C., \& Roulleau-Berger, L. (1995). L'insertion des jeunes en France. Presses Universitaires de France.

Observatoire prospectif du commerce (2016). Repères et tendances - Commerce des articles de sport et équipements de loisirs. Forco (OPCA du commerce et de la distribution).

Pollien, A. (2010). Faire une pause ou bifurquer? Essai de typologie des trajectoires de formation. Éducation \& Sociétés, 26, 123-143. https://doi.org/10.3917/es.026.0123

Rose, J. (1998). Les jeunes face à l'emploi. Desclée de Brouwer.

Silvestre, J-J. (1986). Marchés du travail et crise économique : de la mobilité à la flexibilité. Formation Emploi, 14, 54-61. https://doi.org/10.3406/forem.1986.1173

Tanguy, L. (Eds.) (1986). L'introuvable relation formation-emploi : un état des recherches en France. La Documentation Française.

Tenret, É. (2011). L'école et la méritocratie. Représentations sociales et socialisation scolaire. Presses Universitaires de France.

Verdier, É., \& Vultur, M. (2016). L'insertion professionnelle des jeunes : un concept historique, ambigu et sociétal. Revue Jeunes et Société, 1, 4-28. http://rjs.inrs.ca/index.php/rjs/article/view/67/37

Vernières, M. (1997). L'insertion professionnelle, analyses et débats. Economica.

Vultur, M., \& Trottier, C. (2010). Les trajectoires d'insertion professionnelle des jeunes Québecois, diplômés de l'école secondaire. In C. Papinot \& M. Vultur (Eds.), Les jeunesses au travail (pp. 257-280). Presses de l'Université Laval.

Zimmermann, B. (2011). Ce que travailler veut dire. Une sociologie des capacités et des parcours professionnels. Economica. 